\title{
IMPROVED CULTURE METHODS FOR THE DETECTION OF PS. PYOCYANEA
}

\author{
BY \\ EDWARD J. LOWBURY \\ From the Medical Research Council Burns Unit, Birmingham Accident Hospital
}

(RECEIVED FOR PUblication AUgUST 18, 1950)

Williams, Clayton-Cooper, Faulkner, and Thomas (1944) drew attention to the fact that Pseudomonas pyocyanea may survive and even multiply in solutions of cetrimide (cetyl-trimethyl-ammonium-bromide). The use of this compound in selective media for the identification of Ps. pyocyanea was suggested by Harper and Cawston (1945), who recommended a concentration of $1 \%$ in nutrient agar. This medium, however, was shown by Hood (1948) to inhibit the growth of small inocula of Ps. pyocyanea. Concentrations of cetrimide small enough to be without this inhibitory action allowed the growth of certain strains of Proteus vulgaris and Bacterium coli. The value of enriching $1 \%$ cetrimide agar with serum or blood was therefore tested, and a medium containing $10 \%$ defibrinated horse blood was found satisfactory for dilute inocula of pure cultures and for the examination of swabs from infected burns (Hood, 1948, 1949).

A disadvantage of cetrimide blood agar is its dark-brown colour, which makes it necessary to subculture colonies to a medium (e.g., nutrient agar, or preferably $1 \%$ cetrimide agar) on which $P$ s. pyocyanea can be identified by its pigments. In search of a simpler technique we have studied (1) the use of plasma for enrichment; (2) the use of an ultra-violet lamp for the detection of fluorescence on solid media; and (3) the practical value of lower concentrations of cetrimide without enrichment.

\section{Experimental Methods and Results}

Enrichment of the Selective Medium with Plasma.-In view of the failure of serum previously reported (Hood, 1948), plasma was chosen for trial as an enrichment because it contains components (fibrinogen and probably other substances) which are present in whole blood but not in serum.

Preparation of the Medium.- Hartley's broth, $900 \mathrm{ml}$., containing $2 \%$ of agar was melted; $10 \mathrm{~g}$. of cetrimide ("cetavlon" I.C.I.) were added, and the mixture was allowed to stand in the steamer for one hour. It was then cooled to $55^{\circ} \mathrm{C}$, and $100 \mathrm{ml}$. of citrated horse plasma were added. The medium was poured in 4-in. petri dishes. Cetrimide horse serum agar and cetrimide horse blood agar used for comparison were prepared in the same way. Human plasma was used in some of the observations recorded. (See footnote to Table I.)

Preliminary Tests.-The growth of Ps. pyocyanea on cetrimide plasma agar was tested, using small inocula of 21 strains, 16 of them isolated from infected burns at the Birmingham Accident Hospital, and five kindly supplied to us by Dr. R. Christie, of St. Vincent's Hospital, Melbourne, Australia. Cultures in peptone water were incubated overnight at $37^{\circ} \mathrm{C}$., and $0.02 \mathrm{ml}$. drops of $10^{-5}$ and $10^{-6}$ dilutions in $10 \%$ broth saline were inoculated in quadruplicate 
on the surface of cetrimide plasma agar (C.P.A.) plates. Similar inocula were made on cetrimide serum agar (C.S.A.), on $10 \%$ horse blood agar, and (seven strains only) on $1 \%$ cetrimide $10 \%$ horse blood agar (C.B.A.).

Plates were examined after 24 hours' incubation at $37^{\circ}$ C. Colonies on C.P.A. were smaller than those on blood agar (which were sometimes confluent), and somewhat smaller than those on C.B.A. With few exceptions, colonies of Ps. pyocyanea on C.S.A. were smaller than those on C.P.A., often so small that the use of serum as an enrichment was not pursued. Viable counts on C.P.A. were not significantly different from counts on C.B.A. and on horse blood agar. Comparison of human and horse plasma showed no significant difference in surface viable counts of five strains tested.

Recognition of Ps. pyocyanea on Cetrimide Plasma Agar.-Colonies and confluent growth were recognized by the presence of one or both of the pigments fluorescin and pyocyanin. Confluent growth on C.P.A. almost always showed colour ranging from bluish green to lemon yellow. Separate colonies were often colourless after 24 hours' incubation, but after a further 24 hours most of these were pigmented. Under ultra-violet irradiation (see below) young colonies which were not obviously pigmented often showed characteristic fluorescence.

Suppression of Growth of Bacteria other than Ps. pyocyanea on C.P.A.-Gram-negative bacilli, which are less sensitive to the action of cetrimide than other groups of bacteria, were chosen for the tests. Twenty-one strains of Proteus vulgaris and 15 strains of Bact. coli were heavily inoculated on C.P.A. plates. None of the strains showed any signs of growth after 48 hours' incubation at $37^{\circ} \mathrm{C}$.

Detection of Fluorescence by Ultra-violet Irradiation.-A 125-watt Osira H.P. dark glass mercury vapour lamp was enclosed in the upper part of a wooden box with an opening on one side to admit a culture plate for inspection. The box was kept in a corner of the laboratory sheltered from direct daylight. Colonies and confluent growth of Ps. pyocyanea on blood agar and on C.P.A. plates held 6 in. below the lamp showed brilliant yellow, green, or greenish-blue fluorescence. No other bacterial species showed yellow or green fluorescence, and the faint blue fluorescence of Proteus vulgaris and of some staphylococci on blood agar could usually be distinguished without difficulty. Fluorescence of Ps. pyocyanea on C.B.A. was generally poor (Figs. 1 and 2).

Trial of Cetrimide Plasma Agar and Ultra-violet Irradiation.--It was decided to compare cetrimide plasma agar and cetrimide blood agar as media for the isolation of Ps. pyocyanea from routine swabs taken at dressings of burns. At the same time the value

TABLE I

Comparison of Cetrimide Plasma Agar as a Medium, Ultra-violet Irradiation, AND OTHER METHODS

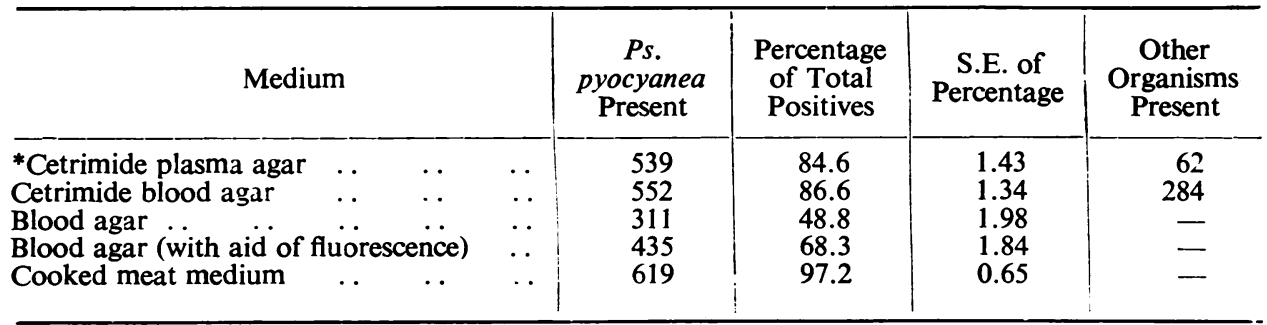

Two thousand and four swabs were examined, and Ps. pyocyanea was isolated in 637.

* One thousand six hundred and thirty-four swabs were plated on horse plasma cetrimide agar, and 370 on a medium containing human plasma. Ninety-three of the swabs in the second group yielded Ps. pyocyanea, including 67 positive on cetrimide blood agar; the difference between these proportions is not significant. 


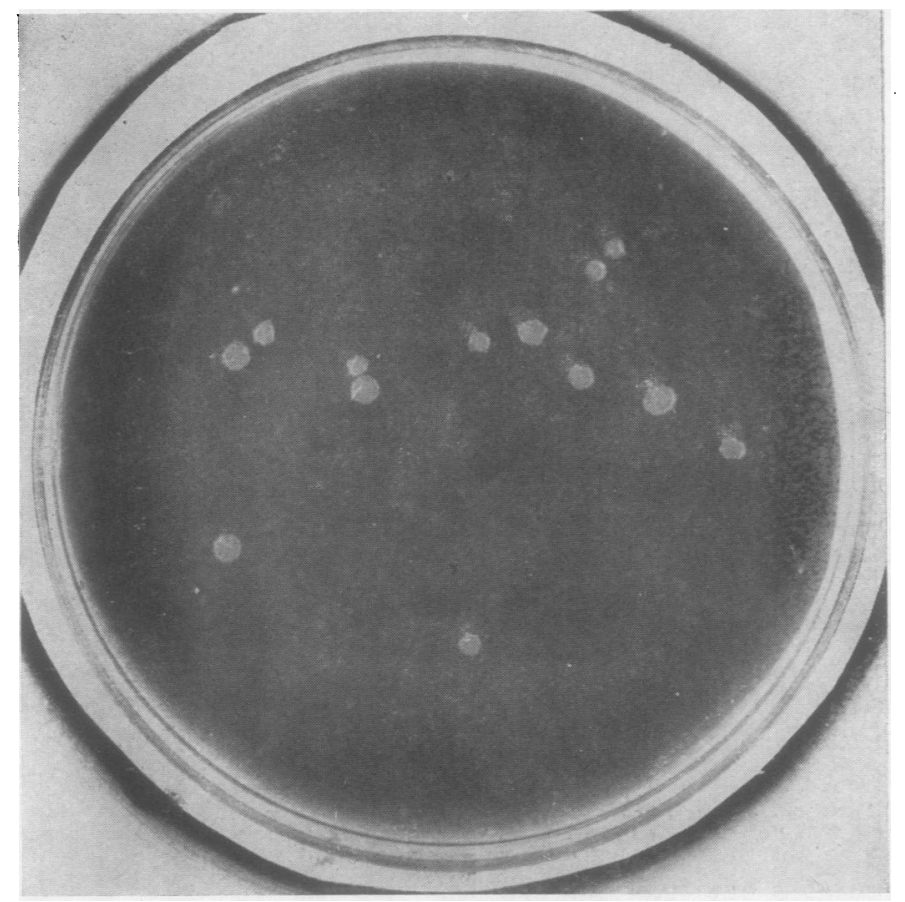

FIG. 1.-Colonies of Ps. pyocyanea photographed by visible light (above) and under the ultra-violet lamp (below). The plate is an air sample on $0.1 \%$ cetrimide agar incubated at $37^{\circ} \mathrm{C}$. for 48 hours.

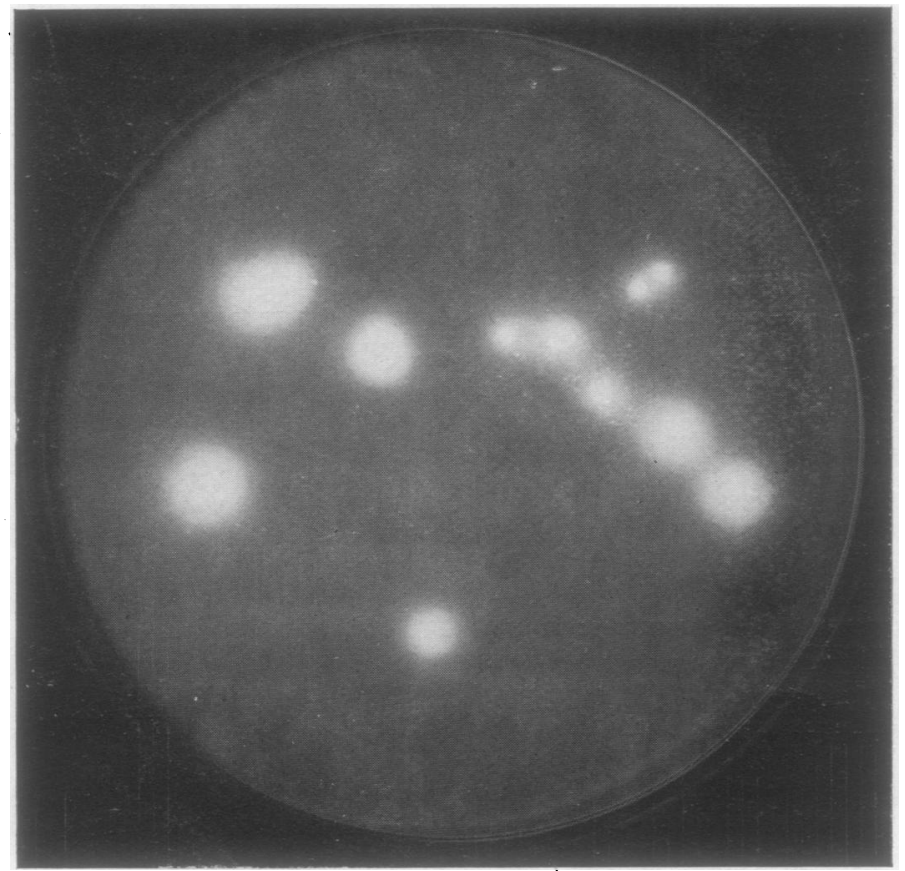

of inspecting culture plates under the $\overrightarrow{\vec{D}}$ ultra-violet lamp was studied in con- $\bar{O}$ junction with other methods used in 흐 the routine bacteriological examination of swabs.

Two thousand and four swabs were inoculated on (1) $10 \%$ horse blood agar (containing 6\% agar to $?$ suppress the swarming of Proteus $\overrightarrow{\vec{\omega}}$ vulgaris); (2) C.B.A. ; (3) C.P.A.; (4) Robertson's cooked meat medium.

Blood agar was inoculated first + and the cooked meat medium last.

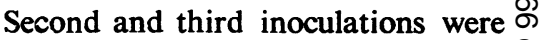
made on C.B.A. and C.P.A., each 은 receiving alternate swabs before the other. Twelve swabs were inoculated ${ }^{\top}$ per plate of each cetrimide medium, without spreading. After overnight incubation at $37^{\circ} \mathrm{C}$. blood agar plates were examined by daylight. $\vec{\theta}$ All colonial types were picked for morphological examination, and 0 Gram-negative bacilli were sub- $\sum^{\circ}$ cultured to plates of $1 \%$ cetrimide agar. Blood agar plates were then examined under the ultra-violet $\stackrel{\odot}{\otimes}$

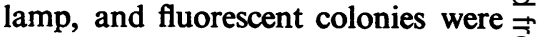
picked to $1 \%$ cetrimide agar (unless $\frac{0}{3}$ characteristic colonies of Ps. pyocyanea had been detected by daylight). Cooked meat cultures and colonies on C.B.A. plates were sub-? cultured to $1 \%$ cetrimide agar. C.P.A. plates were examined by daylight; colonies showing no pigment were $\frac{0}{2}$ spread over a small area, and the plates were reincubated for a further 욱 24 hours, after which they and the $>$ subcultures from blood agar, C.B.A., and cooked meat medium were $N$ examined by daylight and under the ultra-violet lamp.

Growth showing definite green $\mathfrak{\omega}$ pigment on cetrimide agar or cetrimide plasma agar plates incubated $\stackrel{\circ}{\circ}$

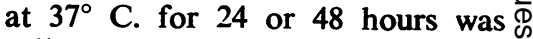
called Ps. pyocyanea. The morpho-? logy of the strains originally isolated from blood agar was checked, and $\underset{\mathbb{D}}{\circ}$ they were all found to be Gram- $\frac{\rho}{\Phi}$ 
negative bacilli. Biochemical reactions of a number of strains were tested and found to be characteristic. Unpigmented growth picked from 38 cetrimide agar plates, by contrast, gave various biochemical and cultural reactions, none of them consistent with those of Ps. pyocyanea.

Table I shows the relative merits of the different media for the isolation of Ps. pyocyanea. Although it received the last inoculation, cooked meat medium gave the highest yield of Ps. pyocyanea (619 out of 637 positive swabs). Conversely, blood agar, though it was inoculated first, showed a consideratly smaller number of cultures positive for $P S$. pyocyanea (311) than any of the other media. C.B.A. and C.P.A. showed higher proporitions of cultures containing Ps. pyocyanea than blood agar (552 and 539 respectively), but this difference was reduced when blood agar plates were examined under the ultra-violet lamp as well as by daylight. (Four hundred and thirty-five were positive.)

C.P.A. and C.B.A. received the only strictly comparable inocula, and the difference between the proportions of swabs positive on these media was not significant (difference between percentages $=2.0$; S.E. of difference of percentages $=1.96$ ). The difference between the percentages positive on blood agar with and without the aid of the fluorescent lamp was significant (difference between percentages $=19.5$; S.E. of difference 2.7).

Practical Value of Lower Concentrations of Cetrimide without Enrichment. - In the course of our studies on the value of plasma as an enrichment it soon became apparent that a rather large proportion of the cultures on C.P.A. which were positive after 48 hours' incubation showed minute colourless colonies and sometimes no recogniz-

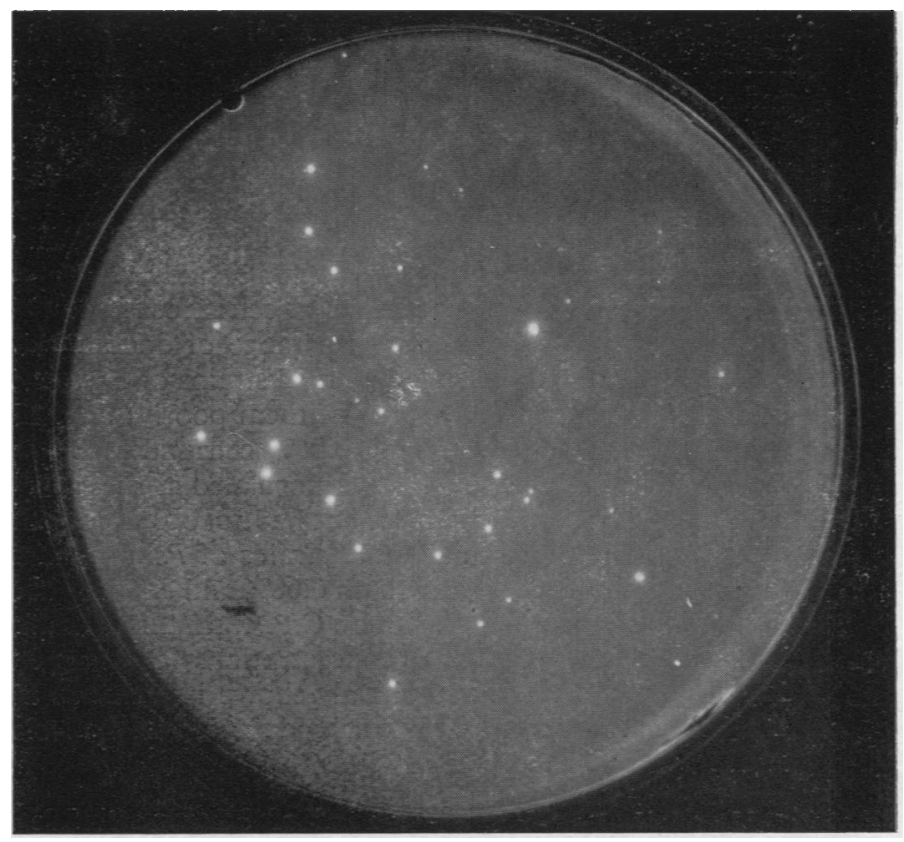

FIG. 2.-Young colonies of $P_{s}$. pyocyanea photographed under the ultra-violet lamp. The plate $(0.1 \%$ cetrimide agar) had been incubated 24 hours at $37^{\circ} \mathrm{C}$. Photograph below shows 24 hours' confluent growth on $0.1 \%$ cetrimide agar of subcultures from cooked meat medium, under the ultra-violet lamp.

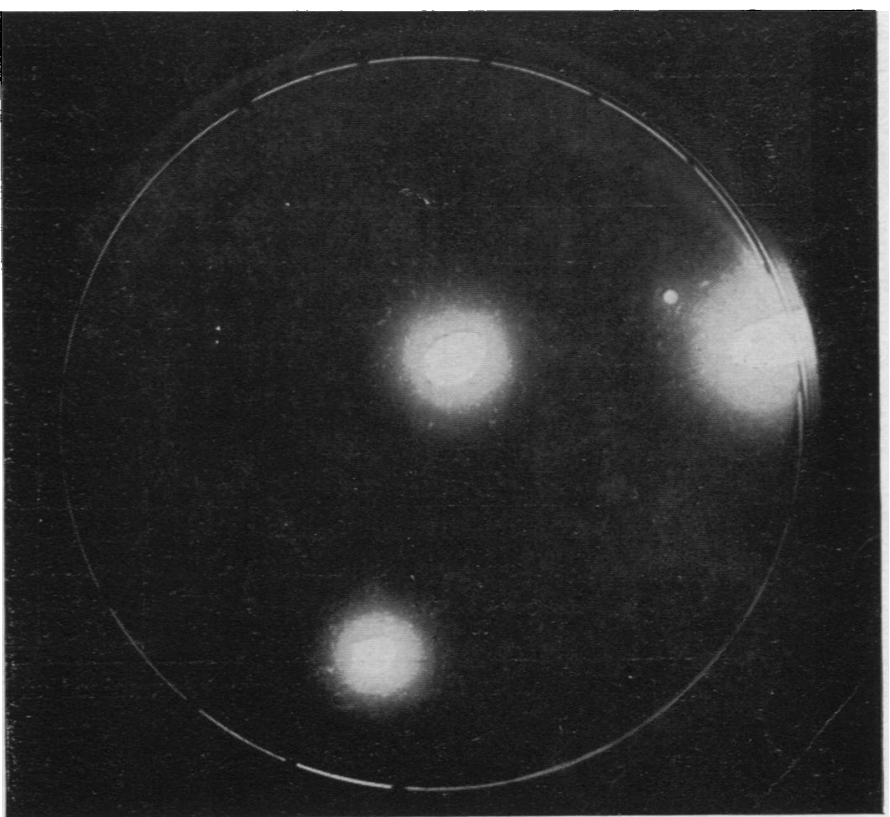


able growth on the day after inoculation. Another fact which came to light (Table $\mathrm{I}$ ) was the presence of coliform bacilli other than Ps. pyocyanea on many plates of C.B.A. (Two hundred and eighty-four cultures on C.B.A. as against 62 cultures on C.P.A. showed the presence of miscellaneous coliforms.) In spite of this, however, C.B.A. was found to be a valuable selective medium, and the objections previously raised against the use of lower concentrations of cetrimide were therefore brought into question. A pale medium less inhibitory to $P s$. pyocyanea than C.P.A. and no less selective than C.B.A. would probably be superior to either. These considerations prompted a study on the merits of unenriched cetrimide agar containing reduced concentrations of cetrimide.

As a preliminary experiment a range of doubling dilutions of cetrimide in agar from $0.03 \%$ to $1.0 \%$ was inoculated with 22 strains of Proteus vulgaris and 14 strains of Bact. coli. Six of these strains grew (three of them poorly) on $0.12 \%$ cetrimide agar. Fifteen strains grew on a medium containing $0.06 \%$ cetrimide. By comparison, 18 of the Proteus and Bact. coli strains grew on C.B.A. inoculated at the same time. Swarming occurred in some strains on $\mathbf{0 . 0 3 \%}$ cetrimide agar, but was not observed in the presence of higher concentrations of cetrimide. Drop counts of 12 strains of Ps. pyocyanea were done on the same range of media and on C.P.A. Growth on $0.06 \%$ and $0.12 \%$ cetrimide agar was richer in quality and in colonial counts than growth on C.P.A. In this test a few strains showed evidence of slight inhibition by cetrimide at all concentrations, including $0.03 \%$ which allowed Proteus vulgaris to swarm. As C.P.A. had already shown its value as a selective medium, however, it was decided that a trial of $0.1 \%$ cetrimide agar for the isolation of Ps. pyocyanea from burns swabs was indicated.

\section{Trial of $0.1 \%$ Cetrimide Agar}

One thousand and fifty-nine swabs from burns were inoculated on $10 \%$ horse blood agar (with $6 \%$ agar), on $0.1 \%$ cetrimide agar (C.A.), on C.P.A., and on cooked meat medium. Cetrimide agar and C.P.A. were made comparable by reversing the order of inoculation of successive swabs on the two media.

Examination of the cultures was done in the way described above for the study of plasma as an enrichment. In addition, plates of C.A. and C.P.A. were examined under the ultra-violet lamp for fluorescence after 24 hours' and 48 hours' incubation.

Table II shows the relative merits of C.A. and C.P.A. Two hundred and eighty-four swabs were found to be positive on C.A. compared with 250 which were positive on C.P.A., indicating a slight but significant advantage in the use of C.A. (difference between the

TABLE II

Comparison of $0.1 \%$ Cetrimide Agar with $1.0 \%$ Cetrimide Plasma Agar

\begin{tabular}{|c|c|c|c|c|c|c|c|c|c|c|}
\hline & & \multicolumn{3}{|c|}{ Growth (Hours) } & \multicolumn{3}{|c|}{$\begin{array}{l}\text { Appearance of } \\
\text { Pigment (Hours) }\end{array}$} & \multicolumn{3}{|c|}{$\begin{array}{c}\text { Appearance of } \\
\text { Fluorescence (Hours) }\end{array}$} \\
\hline & & 24 & 48 & Total & 24 & 48 & Total & 24 & 48 & Total \\
\hline \multirow{2}{*}{$\begin{array}{l}0.1 \% \\
\text { cetrimide } \\
\text { agar }\end{array}$} & Positive ... & 271.0 & 13.0 & 284.0 & 167.0 & 113.0 & 280.0 & 231.0 & 28.0 & 259.0 \\
\hline & $\begin{array}{l}\text { positives } \\
\text { S.E. of } \% \text {.. }\end{array}$ & $\begin{array}{c}77.2 \\
2.25\end{array}$ & 3.7 & $\begin{array}{r}80.9 \\
2.1\end{array}$ & $\begin{array}{l}47.6 \\
2.66\end{array}$ & $\begin{array}{c}32.2 \\
2.49\end{array}$ & $\begin{array}{l}79.8 \\
2.14\end{array}$ & $\begin{array}{c}65.8 \\
2.53\end{array}$ & $\begin{array}{l}7.9 \\
1.47\end{array}$ & $\begin{array}{r}73.7 \\
2.35\end{array}$ \\
\hline \multirow{2}{*}{$\begin{array}{l}1.0 \% \\
\text { cetrimide } \\
\text { plasma } \\
\text { agar }\end{array}$} & Positive ... & 199.0 & 51.0 & 250.0 & 113.0 & 127.0 & 240.0 & 166.0 & 51.0 & 217.0 \\
\hline & $\begin{array}{l}\text { positives } \\
\text { S.E. of } \%\end{array}$ & $\begin{array}{c}56.7 \\
2.63\end{array}$ & $\begin{array}{c}14.5 \\
1.87\end{array}$ & $\begin{array}{r}71.2 \\
2.4\end{array}$ & $\begin{array}{c}32.2 \\
2.49\end{array}$ & $\begin{array}{l}36.2 \\
2.57\end{array}$ & $\begin{array}{c}68.4 \\
2.49\end{array}$ & $\begin{array}{l}47.3 \\
2.66\end{array}$ & $\begin{array}{l}14.5 \\
1.87\end{array}$ & $\begin{array}{c}61.8 \\
2.59\end{array}$ \\
\hline
\end{tabular}

One thousand and fifty-nine swabs were examined and Ps. pyocyanea isolated in 351 . 
percentages $=9.7 ;$ S.E. of difference $=3.2$ ). When the proportions of cultures on the two media which could be identified by pigment after 24 hours' incubation were compared, the superiority of C.A. was more definite (difference between percentages showing pigment after 24 hours $=15.4$; S.E. of difference $=3.65$ ).

The superiority of the cetrimide media to blood agar (197 positives), and their inferiority to cooked meat medium (333 positives), were again demonstrated in this trial.

The practice of examining cetrimide plates by ultra-violet radiation was vindicated. After 24 hours' incubation. 231 cultures on C.A. showed fluorescence, while only 167 showed definite pigment; on C.P.A., 166 cultures were fluorescent after 24 hours' incubation, compared with 113 which were pigmented.

Pigment Production on 0.1\% Cetrimide Agar.-In spite of the greater frequency on C.A. than on C.P.A. of pigmented colonies after 24 hours' incubation, confluent growth on the latter medium was generally found to give better pigment. By the use of a glass electrode it was shown that $1 \%$ cetrimide in distilled water had a $p \mathrm{H}$ of 7.75 , compared with $0.1 \%$ cetrimide in distilled water, which had a $p \mathrm{H}$ of 6.98. It is known that pyocyanin production is inhibited by an acid reaction, in which a red pigment is produced (Wrede, 1930; Young, 1947). Red pigment was observed in some of the cultures on $0.1 \%$ cetrimide agar and occasionally on C.P.A. cultures.

Parallel tests on $0.1 \%$ cetrimide agar prepared without adjusting the $\rho \mathrm{H}$ and on the same medium adjusted to $p \mathrm{H} 7.8$ and $p \mathrm{H} 8.0$ were done, using a number of strains of Ps. pyocyanea. Both green pigmentation and fluorescence were more pronounced at $p \mathrm{H} 7.8$ and $p \mathrm{H} 8.0$ than at $p \mathrm{H} 7.0$. As the medium at $p \mathrm{H} 8.0$ was slightly inhibitory to some strains of $P s$. pyocyanea, $p \mathrm{H} 7.8$ was considered the best reaction for the medium.

\section{Discussion}

The most satisfactory of the selective media under study was arrived at in three stages. First, $1 \%$ cetrimide plasma agar was shown to compare favourably with $1 \%$ cetrimide blood agar in the yield of Ps. pyocyanea from burns swabs plated on the two media; as it was unnecessary to subculture the growth from cetrimide plasma agar for the demonstration of pigment, this medium was adopted for routine purposes. In the second stage, $0.1 \%$ cetrimide agar without enrichment was compared with $1 \%$ cetrimide plasma agar in a similar trial on swabs from burns, and a slight, but significant, advantage was scored by the unenriched $0.1 \%$ cetrimide agar, especially in the yield of positive results after 24 hours' incubation. The advantage of this medium was, however, offset by the poor quality of pigment in confluent growth of Ps. pyocyanea. Adjustment of the medium to $p \mathrm{H} 7.8$ was the third stage, which showed that growth on $0.1 \%$ cetrimide agar could be as green as that on cetrimide plasma agar. As the former medium was more sensitive and apparently no less selective than the latter, it is considered the most satisfactory of the cetrimide media examined.

A combination of methods, such as those described in this paper, is undoubtedly superior to any single method of culture. Fluid media provide the best opportunity for isolating the organism from very small inocula, but they give no indication of the load of infection. Cetrimide media, and particularly $0.1 \%$ cetrimide agar, have shown their superiority to horse blood agar for direct plating of swabs, but may inhibit small inocula of the occasional sensitive strain of Ps. pyocyanea; such strains, however, would almost certainly grow on subculture of the fluid medium inoculated from the same swab. A blood agar plate examined by visible light and under a 
fluorescent lamp provides a quicker result in some cases than either of the selective media studied. As it is possible by this method to recognize Strep. pyogenes, Staph. aureus, Ps. pyocyanea, and other aerobic flora on the same culture plate, it may be recommended for routine purposes when pressure of work precludes the use of more sensitive techniques. Ultra-violet irradiation of blood agar subcultures from liquid media often shows good fluorescence, but this may be obscured by the blue fluorescence of Proteus vulgaris which often accompanies Ps. pyocyanea in burns.

\section{Summary}

Three solid media containing cetrimide were compared with each other and with non-selective methods for the isolation of Ps. pyocyanea from burns swabs. Cetrimide agar, $0.1 \%$, adjusted to $p \mathrm{H} 7.8$ was found to be the most suitable of the selective media. Subculture to a selective medium from cooked meat broth was the most effective method for scanty inocula.

Examination of growth on solid media for fluorescence under an ultra-violet lamp with a dark glass filter was found useful as an aid to the recognition of Ps. pyocyanea.

Acknowledgments are due to Mrs. F. E. Brand and Mr. A. M. Hood for valuable technical assistance.

\section{REFERENCES}

Harper, G. J., and Cawston, W.C. (1945). Bull. Inst. Med. Lab. Tech., 11, 40.

Hood, A. M. (1948). Mon. Bull. Min. Hlth, 7, 248.

(1949). Personal communication.

Williams, R. E. O., Clayton-Cooper, B., Faulkner, H. C., and Thomas, H. E. (1944). Lancet, $1,787$. Wrede, F. (1930). Z. Hyg. InfektKr., 111, 90.

Young, G. (1947). J. Bact., 54, 109. 